\title{
Impaired Metabolomics of Sulfur-Containing Substances in Rats Acutely Treated with Carbon Tetrachloride
}

\author{
Sun Ju Kim', Do Young Kwon', Kwon Hee Choi ${ }^{1}$, Dal Woong Choi ${ }^{2}$ and Young Chul Kim ${ }^{1,3}$ \\ ${ }^{1}$ College of Pharmacy, Seoul National University, Seoul 151-742, Korea \\ ${ }^{2}$ College of Health Science, Korea University, Seoul 136-703, Korea \\ ${ }^{3}$ Research Institute of Pharmaceutical Sciences, Seoul National University, Seoul 151-742, Korea
}

(Received November 12, 2008; Revised November 17, 2008; Accepted November 17, 2008)

\begin{abstract}
Impairment of hepatic metabolism of sulfur-containing amino acids has been known to be linked with induction of liver injury. We determined the early changes in the transsulfuration reactions in liver of rats challenged with a toxic dose of $\mathrm{CCl}_{4}(2 \mathrm{mmol} / \mathrm{kg}$, ip). Both hepatic methionine concentration and methionine adenosyltransferase activity were increased, but S-adenosylmethionine level did not change. Hepatic cysteine was increased significantly from $4 \mathrm{~h}$ after $\mathrm{CCl}_{4}$ treatment. Glutathione (GSH) concentration in liver was elevated in $4 \sim 8 \mathrm{~h}$ and then returned to normal in accordance with the changes in glutamate cysteine ligase activity. Cysteine dioxygenase activity and hypotaurine concentration were also elevated from $4 \mathrm{~h}$ after the treatment. However, plasma GSH concentration was increased progressively, reaching a level at least several fold greater than normal in $24 \mathrm{~h}$. $\gamma$ Glutamyltransferase activity in kidney or liver was not altered by $\mathrm{CCl}_{4}$, suggesting that the increase in plasma GSH could not be attributed to a failure of GSH cycling. The results indicate that acute liver injury induced by $\mathrm{CCl}_{4}$ is accompanied with extensive alterations in the metabolomics of sulfurcontaining amino acids and related substances. The major metabolites and products of the transsulfuration pathway, including methionine, cysteine, hypotaurine, and $\mathrm{GSH}$, are all increased in liver and plasma. The physiological significance of the change in the metabolomics of sulfur-containing substances and its role in the induction of liver injury need to be explored in future studies.
\end{abstract}

Key words: Carbon tetrachloride, Transsulfuration, S-adenosylmethionine, Glutathione, Taurine

\section{INTRODUCTION}

Carbon tetrachloride $\left(\mathrm{CCl}_{4}\right)$ is a model hepatotoxicant. The induction of $\mathrm{CCl}_{4}$ toxicity is attributed to generation of a free radical, $\cdot \mathrm{CCl}_{3}$, by enzyme reactions mediated mostly by cytochrome P450 2E1. The free radical binds covalently to lipids and proteins, resulting

Correspondence to: Young Chul Kim, College of Pharmacy, Seoul National University, San 56-1 Shinrim-Dong, Kwanak$\mathrm{Ku}$, Seoul 151-742, Korea

E-mail: youckim@snu.ac.kr

List of Abbreviations: SAM, S-adenosylmethionine; MAT, methionine adenosyltransferase; SAH, S-adenosylhomocysteine; C $\beta S$, cystathionine $\beta$-synthase; $C \gamma \mathrm{L}$, cystathionine $\gamma$ lyase; $\mathrm{GSH}$, glutathione; CDO, cysteine dioxygenase; GCL, glutamate cysteine ligase; AST, aspartate aminotransferase; ALT, alanine aminotransferase; $\mathrm{sCr}$, serum creatinine; BUN, blood urea nitrogen; MDA, malondialdehyde; GGT, $\gamma$-glutamyltransferase; ER, endoplasmic reticulum; LPS, lipopolysaccharide; ANIT, $\alpha$-naphthylisothiocyanate; GSSG, glutathione disulfide; MRPs, multidrug resistance proteins. in structural damage of membranes and a variety of enzymes. The reactive species also initiates lipid peroxidation by attacking enoic fatty acids leading to generation of organic free radicals, which may in turn react with $\mathrm{O}_{2}$ to form peroxides and other cytotoxic metabolites (Recknagel et al., 1989).

In mammals the liver plays a central role in the sulfuramino acid metabolism (Mudd and Poole, 1975). Sulfuramino acid metabolism occurs primarily via the transsulfuration pathway, which results in transfer of sulfur from methionine to serine to form cysteine. The first step in methionine metabolism is formation of S-adenosylmethionine (SAM) that is catalyzed by methionine adenosyltransferase (MAT). SAM serves as a methyl donor for various biological methylation reactions, and the coproduct of transmethylation, S-adenosylhomocysteine $(\mathrm{SAH})$, is hydrolyzed to yield homocysteine which is either remethylated to methionine or condensed with serine into cystathionine. Condensation of homocysteine and serine to cystathionine and subsequent release of 
cysteine from cystathionine are mediated by cystathionine $\beta$-synthase $(C \beta S)$ and cystathionine $\gamma$-lyase $(C \gamma L)$, respectively. Cysteine is metabolized in liver to yield either taurine, inorganic sulfate, or glutathione (GSH). Cysteine dioxygenase (CDO) catalyzes oxidation of this amino acid to cysteinesulfinate that is converted to taurine via hypotaurine. Synthesis of GSH from cysteine is mediated by glutamate cysteine ligase (GCL) and GSH synthetase, consecutively.

The intermediates or final products in the transsulfuration reactions, including SAM and GSH, have essential roles in the maintenance of the normal biochemistry and physiology of mammalian livers. In this study we examined the changes in hepatic metabolism of sulfuramino acids in acute liver injury induced by $\mathrm{CCl}_{4}$ treatment. Despite the well-known hepatotoxic potential of this organic solvent, its effects on the hepatic transsulfuration reactions have hardly been studied. Considering the devastating effects of $\mathrm{CCl}_{4}$ on the liver, it is suspected that significant alterations in the metabolomics of sulfur-containing substances would take place, which may encompass important toxicological implications in liver. Since the hepatotoxicity of $\mathrm{CCl}_{4}$ is primarily associated with lipid peroxidation, examination of its effects on the transsulfuration pathway that supplies GSH, a major antioxidant in liver, as the final product was of particular interest.

\section{MATERIALS AND METHODS}

Animals and treatments. Male Sprague-Dawley rats were purchased from Dae-Han Laboratory Animal (Seoul, Korea). The use of animals was in compliance with the guidelines established by the Animal Care Committee of this institute. Rats were housed in temperature $\left(22 \pm 2^{\circ} \mathrm{C}\right)$ and humidity $(55 \pm 5 \%)$ controlled rooms with a $12 \mathrm{~h}$ light/dark cycle. Laboratory rat chow and tap water were allowed ad libitum. Rats, weighing $250 \sim 300 \mathrm{~g}$, were fasted from $24 \mathrm{~h}$ before $\mathrm{CCl}_{4}$ treatment. $\mathrm{CCl}_{4}$ diluted in corn oil was administered intraperitoneally to rats at a dose of $2 \mathrm{mmol} / \mathrm{kg}$. Control rats received an equivalent volume of corn oil.

Determination of liver and kidney toxicity. Blood was sampled from abdominal aorta in rats under light ether anesthesia. Activities of aspartate aminotransferase (AST) and alanine aminotransferase (ALT) in serum were determined by the method of Reitman and Frankel (1957). Serum creatinine ( $\mathrm{sCr}$ ) and blood urea nitrogen (BUN) were measured using commercially available kits (Youngdong Pharmaceutical, Seoul, Korea).
Malondialdehyde (MDA) in liver was quantified using the method of Volpi and Tarugi (1998). A HPLC system equipped with a fluorescence detector and a $5 \mu \mathrm{m}$ Symmetry C18 reversed phase column $(4.6 \times 150 \mathrm{~mm}$; Eka, Bohus, Sweden) was used. The mobile phase was composed of $35 \%$ methanol and $65 \% 50 \mathrm{mM}$ sodium phosphate buffer, $\mathrm{pH} 7.0$.

Determination of sulfur-containing metabolites. Liver was homogenized in a fourfold volume of cold 1 $M$ perchloric acid. After centrifugation, GSH concentration in the supernatant was determined using an enzymatic recycling method (Griffith, 1980). Cysteine level was estimated by the acid-ninhydrin method (Gaitonde, 1967). The method of She et al. (1994) was employed to determine SAM and SAH concentrations. The supernatant was directly applied to a HPLC system with a UV detector and a TSK-GEL ODS-80TM column (4.6 $\times$ $250 \mathrm{~mm}$ ) (Tosoh, Tokyo, Japan).

Liver was homogenized in a fivefold volume of cold methanol for analysis of methionine, hypotaurine and taurine. Serum was diluted with methanol. Methionine, hypotaurine and taurine were derivatized with $O$ phthalaldehyde/2-mercaptoethanol and quantified using HPLC with a fluorescence detector and a $3.5 \mu \mathrm{m}$ Kromasil C18 column $(4.6 \times 100 \mathrm{~mm})$ (Eka, Bohus, Sweden). Methionine was measured using the method of Rajendra (1987). The method of Ide (1997) was used to separate hypotaurine and taurine.

Enzyme assays. Liver homogenate in a threefold volume of ice-cold buffer consisting of $0.154 \mathrm{M} \mathrm{KCl} /$ $50 \mathrm{mM}$ Tris- $\mathrm{HCl}$ and $1 \mathrm{mM}$ EDTA (pH 7.4) was centrifuged at $10,000 \mathrm{~g}$ for $20 \mathrm{~min}$. The supernatant was further centrifuged at $104,000 \mathrm{~g}$ for $60 \mathrm{~min}$. The $104,000 \mathrm{~g}$ supernatant fraction (cytosol) was used to determine the enzyme activities.

The activity of MAT was estimated by quantifying SAM and SAH production as described above. The reaction mixture consisted of $80 \mathrm{mM}$ Tris- $\mathrm{HCl} / 50 \mathrm{mM} \mathrm{KCl}(\mathrm{pH}$ 7.4), $5 \mathrm{mM}$ ATP, $40 \mathrm{mM} \mathrm{MgCl}_{2}, 0.1 \mathrm{mM}$ methionine, and enzyme solution containing $1 \mathrm{mg}$ protein in a final volume of $1 \mathrm{ml}$. The incubation was carried at $37^{\circ} \mathrm{C}$ for 30 min. $C \beta S$ activity was determined by quantifying generation of cystathionine (Kashiwamata and Greenberg, 1970). The method of Matsuo and Greenberg (1957) was used to determine $\mathrm{C} \gamma \mathrm{L}$ activity. GCL activity was determined using the method of Sekura and Meister (1977). Formation of $\gamma$-glutamylcysteine was quantified after O-phthalaldehyde derivatization according to a HPLC separation/fluorometric detection method (Yan and Huxtable, 1995). The method of Bagley et al. 
(1995) was used to measure CDO activity. The amount of cysteinesulfinate formed was quantified by a HPLC system equipped with a fluorescence detector and a $3.5 \mu \mathrm{m}$ Kromasil C18 column $(4.6 \times 100 \mathrm{~mm})$ after Ophthalaldehyde/2-mercaptoethanol derivatization.

The activity of $\gamma$-glutamyltransferase (GGT) was measured using whole tissue homogenates. Renal GGT activity was assayed kinetically at $37^{\circ} \mathrm{C}$ by the method of Orlowski and Meister (1963). $\gamma$-Glutamyl-p-nitroanilide was used as a substrate. A non-kinetic method was used to determine hepatic GGT activity (Hinchman and Ballatori, 1999). Tissue homogenates and the substrate were added to a reaction mixture containing Tris-glycine buffer $(\mathrm{pH} 8.0)$ for incubation at $37^{\circ} \mathrm{C}$ for $30 \mathrm{~min}$.

Data analysis. All results expressed as the mean \pm S.E.M. were analyzed by a two-tailed Student's $t$-test. The acceptable level of significance was established at $P<0.05$ except when otherwise indicated.

\section{RESULTS}

$\mathrm{CCl}_{4}$-induced liver and kidney toxicity. Serum AST and ALT activities were elevated significantly from $4 \mathrm{~h}$ following $\mathrm{CCl}_{4}$ treatment (Table 1). The enzyme activities in serum were increased steadily for $24 \mathrm{~h}$ after the treatment. Hepatic MDA levels were increased rapidly after $\mathrm{CCl}_{4}$ treatment, but returned to normal at $\mathrm{t}=$ $8 \mathrm{~h}$. The increase in lipid peroxidation preceded the elevation of serum AST and ALT activities, suggesting a causal role of lipid peroxidation in $\mathrm{CCl}_{4}$-induced hepatotoxicity. The dose of $\mathrm{CCl}_{4}$ used in this study also induced renal injury. Both BUN and $\mathrm{sCr}$ were elevated at $\mathrm{t}=8 \mathrm{~h}$, which remained at a level higher than control till $24 \mathrm{~h}$ after $\mathrm{CCl}_{4}$ treatment.

Concentration of sulfur-containing metabolites in liver. Changes in hepatic concentrations of sulfur-containing substances are summarized in Table 2. Methionine was not changed immediately after $\mathrm{CCl}_{4}$ treatment,

Table 1. Changes in parameters of liver and kidney toxicity in rats treated with $\mathrm{CCl}_{4}$

\begin{tabular}{|c|c|c|c|c|c|c|}
\hline \multirow{2}{*}{ Time } & \multirow{2}{*}{ Group } & ALT & AST & \multirow{2}{*}{$\begin{array}{c}\text { MDA } \\
\text { nmol/g liver }\end{array}$} & $\mathrm{sCr}$ & BUN \\
\hline & & \multicolumn{2}{|c|}{ units $/ \mathrm{ml}$ serum } & & \multicolumn{2}{|c|}{$\mathrm{mg} / \mathrm{dl}$ serum } \\
\hline $\mathrm{Oh}$ & Control & $34.5 \pm 2.0$ & $79.9 \pm 1.0$ & $3.34 \pm 0.36$ & $0.30 \pm 0.02$ & $19.1 \pm 1.1$ \\
\hline \multirow{2}{*}{$2 \mathrm{~h}$} & Vehicle & $34.4 \pm 2.2$ & $95.1 \pm 7.5$ & $3.71 \pm 0.26$ & $\mathrm{~N} / \mathrm{D}$ & N/D \\
\hline & $\mathrm{CCl}_{4}$ & $36.4 \pm 2.4$ & $112.3 \pm 7.5$ & $5.82 \pm 0.25^{* *}$ & N/D & N/D \\
\hline \multirow{2}{*}{$4 \mathrm{~h}$} & Vehicle & $33.1 \pm 3.1$ & $89.2 \pm 7.7$ & $4.45 \pm 0.17$ & N/D & N/D \\
\hline & $\mathrm{CCl}_{4}$ & $273.2 \pm 39.4^{* *}$ & $384.5 \pm 48.9^{* *}$ & $5.91 \pm 0.35^{\star *}$ & $N / D$ & N/D \\
\hline \multirow{2}{*}{$8 \mathrm{~h}$} & Vehicle & $32.2 \pm 3.0$ & $88.5 \pm 6.5$ & $4.84 \pm 0.23$ & $0.31 \pm 0.02$ & $16.9 \pm 0.9$ \\
\hline & $\mathrm{CCl}_{4}$ & $629.8 \pm 135.8^{* *}$ & $596.2 \pm 96.5^{* *}$ & $4.93 \pm 0.21$ & $0.46 \pm 0.05^{*}$ & $19.9 \pm 0.7^{*}$ \\
\hline \multirow{2}{*}{$24 \mathrm{~h}$} & Vehicle & $32.8 \pm 4.4$ & $79.8 \pm 5.5$ & $5.31 \pm 0.36$ & $0.30 \pm 0.03$ & $16.1 \pm 0.6$ \\
\hline & $\mathrm{CCl}_{4}$ & $1564.9 \pm 177.8^{* *}$ & $1980.6 \pm 397.2^{* *}$ & $4.92 \pm 0.19$ & $0.41 \pm 0.03^{*}$ & $22.2 \pm 2.1^{*}$ \\
\hline
\end{tabular}

Rats were treated intraperitoneally with $\mathrm{CCl}_{4}(2 \mathrm{mmol} / \mathrm{kg})$. Each value represents mean \pm S.E.M. for 4 or 5 rats. ${ }^{*},{ }^{* *}$ Significantly different from the rats treated with vehicle only at $P<0.05,0.01$, respectively. N/D; not determined.

Table 2. Changes in sulfur-containing substances in liver of rats treated with $\mathrm{CCl}_{4}$

\begin{tabular}{|c|c|c|c|c|c|c|c|c|}
\hline \multirow{2}{*}{ Time } & \multirow{2}{*}{ Group } & Methionine & SAM & SAH & Cysteine & GSH & Hypotaurine & Taurine \\
\hline & & \multicolumn{4}{|c|}{$\mathrm{nmol} / \mathrm{g}$ liver } & \multicolumn{3}{|c|}{$\mu \mathrm{mol} / \mathrm{g}$ liver } \\
\hline $\mathrm{Oh}$ & Control & $64.0 \pm 3.4$ & $52.1 \pm 2.6$ & $23.9 \pm 2.3$ & $109 \pm 4.4$ & $4.42 \pm 0.09$ & $0.22 \pm 0.02$ & $1.95 \pm 0.28$ \\
\hline \multirow{2}{*}{$2 \mathrm{~h}$} & Vehicle & $64.8 \pm 5.5$ & $53.1 \pm 2.5$ & $23.5 \pm 1.6$ & $100 \pm 9.6$ & $4.42 \pm 0.29$ & $0.20 \pm 0.03$ & $5.20 \pm 1.15$ \\
\hline & $\mathrm{CCl}_{4}$ & $73.8 \pm 4.3$ & $52.2 \pm 3.3$ & $21.8 \pm 0.4$ & $87 \pm 2.9$ & $4.16 \pm 0.09$ & $0.31 \pm 0.07$ & $4.70 \pm 0.84$ \\
\hline \multirow{2}{*}{$4 \mathrm{~h}$} & Vehicle & $66.7 \pm 2.2$ & $48.6 \pm 3.3$ & $22.4 \pm 1.1$ & $85 \pm 1.5$ & $3.44 \pm 0.11$ & $0.16 \pm 0.03$ & $4.29 \pm 0.55$ \\
\hline & $\mathrm{CCl}_{4}$ & $77.3 \pm 4.5$ & $55.2 \pm 1.1$ & $19.2 \pm 0.8$ & $109 \pm 4.8^{* *}$ & $4.79 \pm 0.19^{* *}$ & $0.32 \pm 0.03^{* *}$ & $2.61 \pm 0.41^{*}$ \\
\hline \multirow{2}{*}{$8 \mathrm{~h}$} & Vehicle & $72.2 \pm 6.4$ & $53.1 \pm 2.2$ & $24.3 \pm 1.5$ & $87 \pm 3.2$ & $3.43 \pm 0.13$ & $0.14 \pm 0.02$ & $5.31 \pm 0.87$ \\
\hline & $\mathrm{CCl}_{4}$ & $74.6 \pm 2.2$ & $54.5 \pm 1.4$ & $17.2 \pm 0.9^{* *}$ & $108 \pm 4.3^{* *}$ & $6.22 \pm 0.26^{* *}$ & $0.19 \pm 0.03$ & $1.92 \pm 0.32^{* *}$ \\
\hline \multirow{2}{*}{$24 \mathrm{~h}$} & Vehicle & $54.5 \pm 5.1$ & $53.3 \pm 2.1$ & $20.9 \pm 0.9$ & $86 \pm 6.7$ & $3.95 \pm 0.20$ & $0.09 \pm 0.01$ & $2.62 \pm 0.61$ \\
\hline & $\mathrm{CCl}_{4}$ & $92.1 \pm 3.5^{\star *}$ & $57.4 \pm 1.5$ & $17.8 \pm 1.1$ & $156 \pm 9.6^{\star *}$ & $4.52 \pm 0.26$ & $0.33 \pm 0.10^{*}$ & $2.13 \pm 0.08$ \\
\hline
\end{tabular}

Each value represents mean \pm S.E.M. for 4 or 5 rats. ${ }^{*},{ }^{*}$ Significantly different from the rats treated with vehicle only at $P<0.05$, 0.01 , respectively. 
Table 3. Changes in sulfur-containing substances in plasma of rats treated with $\mathrm{CCl}_{4}$

\begin{tabular}{clllll}
\hline \hline \multirow{2}{*}{ Time } & \multirow{2}{*}{ Group } & Methionine & \multicolumn{2}{c}{ Cysteine } & \multicolumn{2}{c}{ GSH } \\
\cline { 3 - 6 } & & \multicolumn{4}{c}{$\mathrm{nmol} / \mathrm{ml}$ plasma } \\
\hline \multirow{2}{*}{$\mathrm{Oh}$} & Control & $51.9 \pm 1.9$ & $12.5 \pm 0.4$ & $3.54 \pm 0.27$ & $167 \pm 14$ \\
\hline \multirow{2}{*}{$2 \mathrm{~h}$} & Vehicle & $54.0 \pm 1.5$ & $12.6 \pm 1.1$ & $3.43 \pm 0.19$ & $144 \pm 20$ \\
& $\mathrm{CCl}_{4}$ & $52.9 \pm 2.4$ & $10.1 \pm 1.0$ & $3.27 \pm 0.18$ & $125 \pm 11$ \\
\hline \multirow{2}{*}{$4 \mathrm{~h}$} & Vehicle & $52.1 \pm 2.4$ & $11.3 \pm 0.6$ & $3.19 \pm 0.29$ & $157 \pm 12$ \\
& $\mathrm{CCl}_{4}$ & $57.5 \pm 3.8$ & $8.9 \pm 0.4^{*}$ & $5.23 \pm 0.38^{* *}$ & $238 \pm 11^{* *}$ \\
\hline \multirow{2}{*}{$8 \mathrm{~h}$} & Vehicle & $41.6 \pm 2.0$ & $10.7 \pm 1.1$ & $2.75 \pm 0.14$ & $148 \pm 15$ \\
& $\mathrm{CCl}_{4}$ & $48.5 \pm 1.5^{*}$ & $11.9 \pm 0.7$ & $8.52 \pm 1.49^{* *}$ & $179 \pm 4$ \\
\hline \multirow{2}{*}{$24 \mathrm{~h}$} & $V^{*}$ & $48.2 \pm 1.1$ & $8.1 \pm 0.7$ & $3.20 \pm 0.37$ & $177 \pm 27$ \\
& $\mathrm{CCl}_{4}$ & $73.0 \pm 1.5^{* *}$ & $13.5 \pm 1.2^{* *}$ & $30.95 \pm 3.55^{* *}$ & $299 \pm 42^{*}$ \\
\hline
\end{tabular}

Each value represents mean \pm S.E.M. for 4 or 5 rats. ${ }^{*},{ }^{* *}$ Significantly different from the rats treated with vehicle only at $P<0.05$, 0.01 , respectively.

Table 4. Changes in enzyme activities involved in the sulfur-containing amino acid metabolism in liver of rats treated with $\mathrm{CCl}_{4}$

\begin{tabular}{|c|c|c|c|c|c|c|c|c|}
\hline \multirow[b]{2}{*}{ Time } & \multirow[b]{2}{*}{ Group } & MAT & C $\mathrm{C} \beta S$ & $\mathrm{C}_{\gamma \mathrm{L}}$ & $\overline{\mathrm{GCL}}$ & CDO & \multicolumn{2}{|c|}{ GGT } \\
\hline & & \multicolumn{5}{|c|}{ product formed $(\mathrm{nmol}) / \mathrm{min} / \mathrm{mg}$ protein } & $\begin{array}{c}\mathrm{nmol} / \mathrm{min} / \mathrm{g} \\
\text { liver }\end{array}$ & $\begin{array}{c}\mu \mathrm{mol} / \mathrm{min} / \mathrm{g} \\
\mathrm{kidney}\end{array}$ \\
\hline $\mathrm{Oh}$ & Control & $0.18 \pm 0.01$ & $6.94 \pm 0.27$ & $68.43 \pm 3.45$ & $5.57 \pm 0.22$ & $0.029 \pm 0.001$ & $163 \pm 22$ & $259 \pm 5$ \\
\hline \multirow{2}{*}{$2 \mathrm{~h}$} & Vehicle & $0.20 \pm 0.02$ & $8.47 \pm 0.51$ & $73.03 \pm 5.87$ & $6.53 \pm 0.65$ & $0.027 \pm 0.002$ & N/D & N/D \\
\hline & $\mathrm{CCl}_{4}$ & $0.20 \pm 0.02$ & $6.21 \pm 0.52^{*}$ & $66.48 \pm 1.19$ & $7.22 \pm 0.98$ & $0.026 \pm 0.001$ & N/D & N/D \\
\hline \multirow{2}{*}{$4 \mathrm{~h}$} & Vehicle & $0.32 \pm 0.03$ & $6.38 \pm 0.48$ & $70.11 \pm 2.78$ & $6.93 \pm 0.57$ & $0.030 \pm 0.003$ & N/D & N/D \\
\hline & $\mathrm{CCl}_{4}$ & $0.56 \pm 0.02^{* *}$ & $6.39 \pm 0.72$ & $69.41 \pm 2.97$ & $8.70 \pm 0.61$ & $0.057 \pm 0.005^{\star *}$ & N/D & N/D \\
\hline \multirow{2}{*}{$8 \mathrm{~h}$} & Vehicle & $0.50 \pm 0.05$ & $6.30 \pm 0.30$ & $60.57 \pm 2.13$ & $6.38 \pm 0.66$ & $0.028 \pm 0.002$ & $171 \pm 16$ & $273 \pm 6$ \\
\hline & $\mathrm{CCl}_{4}$ & $1.11 \pm 0.21^{*}$ & $5.79 \pm 0.56$ & $57.70 \pm 2.23$ & $10.45 \pm 0.47^{* *}$ & $0.040 \pm 0.002^{* *}$ & $166 \pm 31$ & $263 \pm 4$ \\
\hline \multirow{2}{*}{$24 \mathrm{~h}$} & Vehicle & $0.19 \pm 0.01$ & $5.29 \pm 0.22$ & $69.84 \pm 1.18$ & $7.00 \pm 0.21$ & $0.023 \pm 0.001$ & $117 \pm 15$ & $256 \pm 7$ \\
\hline & $\mathrm{CCl}_{4}$ & $0.27 \pm 0.03^{*}$ & $5.41 \pm 0.24$ & $44.06 \pm 2.74^{* *}$ & $7.09 \pm 0.66$ & $0.022 \pm 0.001$ & $114 \pm 10$ & $241 \pm 7$ \\
\hline
\end{tabular}

Each value represents mean \pm S.E.M. for 4 or 5 rats. ${ }^{*},{ }^{* *}$ Significantly different from the rats treated with vehicle only at $P<0.05$, 0.01 , respectively. N/D; not determined.

but increased significantly at $t=24 \mathrm{~h}$. SAM and $S A H$ levels appeared to be unaffected by the dose of $\mathrm{CCl}_{4}$ administered. Cysteine was elevated gradually from $4 \mathrm{~h}$ after the treatment. Major metabolic products of cysteine catabolism, GSH and hypotaurine, were also elevated in liver from $t=4 \mathrm{~h}$. On the contrary, hepatic taurine was decreased significantly from $t=4$ to $8 \mathrm{~h}$.

Plasma methionine and cysteine levels were changed in a manner similar to that shown in liver (Table 3). However, GSH levels in plasma, elevated from $4 \mathrm{~h}$ after the treatment, increased progressively, reaching approximately a tenfold of control in $24 \mathrm{~h}$. Taurine was elevated from $4 \mathrm{~h}$ following $\mathrm{CCl}_{4}$ treatment, although the magnitude of increase was much less than that of GSH.

Enzyme activities involved in the transsulfuration reactions. Hepatic MAT activity was increased rapidly by $\mathrm{CCl}_{4}$ treatment, which remained at a level significantly higher than control for $24 \mathrm{~h}$ (Table 4). MAT activ- ity in control rats was changed significantly in $24 \mathrm{~h}$, suggesting a circadian variation of this enzyme. $C \beta S$ and $\mathrm{C} \gamma \mathrm{L}$ activities appeared to be slightly decreased. The activities of GCL and CDO, which have key roles in the conversion of cysteine to GSH and hypotaurine, were induced transiently, but significantly, and returned to normal in $24 \mathrm{~h}$. GGT activity was greater in kidney than in liver by more than a thousand-fold. Neither the liver GGT activity nor the renal GGT activity was altered by a hepatotoxic dose of $\mathrm{CCl}_{4}$.

\section{DISCUSSION}

It has long been realized that chronic liver injury is often associated with impairment of sulfur-amino acid metabolism (Kinsell et al., 1947; Horowitz et al., 1981). A link between ethanol-induced elevation of homocysteine and endoplasmic reticulum (ER) stress has also been demonstrated ( $\mathrm{Ji}$ et al., 2004). Further studies 
have shown that an elevation of homocysteine in liver may trigger activation of hepatic stellate cells through oxidative stress, which ultimately results in hepatic fibrosis (Adinolfi et al., 2005). Conversely, metabolic intermediates or products in the transsulfuration reactions, such as SAM, S-methylthioadenosine and taurine, were shown to be effective against induction of hepatic fibrosis in experimental animals (Simile et al., 2001; Nieto and Cederbaum, 2005; Miyazaki et al., 2005). This suggests a possibility that disturbance of the metabolomics of sulfur-containing substances in liver has an important role in the development of chronic hepatic injury. In fact we observed that induction of oxidative stress-mediated liver injury by a hepatotoxicant, such as ethanol, bacterial lipopolysaccharide (LPS), $\alpha$-naphthylisothiocyanate (ANIT), chloroform, and high-fat diet, was all accompanied with profound changes in the hepatic transsulfuration reactions (Kim and Kim, 2002, 2005; $\mathrm{Kim}$ et al., 2005, 2008; Kwon et al., 2008). The recovery from liver injury also corresponded with retrieval of hepatic levels of sulfur-containing substances.

The present study was conducted to characterize the early changes in hepatic metabolism of sulfur-containing substances in rats treated with an acute dose of $\mathrm{CCl}_{4}$, a model hepatotoxicant frequently used to develop liver injury in animal experiments. A toxic dose of $\mathrm{CCl}_{4}$ increased the activity of MAT rapidly. This is contrary to the report showing a reduction of MAT activity in rats treated with $\mathrm{CCl}_{4}$ repeatedly (Corrales et al., 1992). It has been suggested that MAT activity is regulated by cellular redox state in liver. Hepatic MAT activity was inhibited by addition of GSSG (Pajares et al., 1992) and also by hydroxyl radical (Sánchez-Góngora et al., 1997). Inactivation of MAT was reversed by GSH and other thiol-reducing agents (Corrales et al., 1999). In this study, hepatic MAT activity was increased significantly from $4 \mathrm{~h}$ after $\mathrm{CCl}_{4}$ treatment, which might be associated with the elevation of GSH levels in liver. However, the induction of MAT activity did not result in an increase in SAM levels. In addition to its role as an intermediate in the transsulfuration reactions, SAM is the principal biological methyl donor and also a provider of aminopropyl groups utilized in polyamine synthesis. Therefore, SAM is not only an intermediate metabolite in methionine catabolism, but also acts as an intracellular control switch that regulates essential hepatic functions such as regeneration, differentiation, and the sensitivity of this organ to injury (Mato and Lu, 2007). The present results suggest that SAM, although its synthesis might be enhanced via induction of MAT activity and methionine availability, reduced in liver due to rapid consumption in a metabolic reaction(s) associated with induction of acute liver injury and/or regeneration process resulting from $\mathrm{CCl}_{4}$ intoxication.

Hepatic GSH levels were elevated significantly from $4 \mathrm{~h}$ after $\mathrm{CCl}_{4}$ treatment. Synthesis of GSH in liver is limited mostly by two factors; the availability of cysteine and the activity of GCL that catalyzes a rate limiting step in GSH synthesis (Lu, 1998). Hepatic cysteine was increased from $4 \mathrm{~h}$ following $\mathrm{CCl}_{4}$ treatment, and remained at a level significantly higher than normal in this study. The GCL activity was also increased after $\mathrm{CCl}_{4}$ treatment. Thus, the elevation of hepatic GSH levels was attributed to the enhanced generation of this tripeptide in hepatocytes.

In liver the cysteine concentration is tightly regulated by a balance between the rates of its synthesis, hepatic uptake from blood, and metabolism to $\mathrm{GSH}$, inorganic sulfate and taurine (Stipanuk et al., 1992). The dose of $\mathrm{CCl}_{4}$ administered to rats did not increase either $\mathrm{C} \beta \mathrm{S}$ or $\mathrm{C} \gamma \mathrm{L}$ activity in this study. Therefore, the elevated cysteine levels may not be accounted for by the enhancement of its synthesis in liver. The hepatic levels of GSH and hypotaurine, the major metabolic products from cysteine, were increased by $\mathrm{CCl}_{4}$ treatment, excluding a possibility that inhibition of cysteine catabolism to either GSH or taurine could be responsible for the increment of this sulfur-amino acid. Recently we observed that a toxic dose of $\mathrm{CCl}_{4}$ elevated the hepatic cysteine contents in mice as well (Lee and Kim, 2007). The mechanism of cysteine elevation in liver remains unclear, but it seems that an increase in its uptake from plasma plays a role here. This view appears to be supported by the transient, but significant decrease in cysteine levels in plasma at $4 \mathrm{~h}$ following $\mathrm{CCl}_{4}$ treatment.

Cysteine is catabolized oxidatively to cysteinesulfinate, which is introduced into several pathways including taurine synthesis, sulfate production, and generation of pyruvate. In the present study hepatic cysteine levels and $\mathrm{CDO}$ activity were elevated from $4 \mathrm{~h}$ after $\mathrm{CCl}_{4}$ treatment, which was accompanied with an increase in hypotaurine. The induction of CDO activity is in agreement with the suggestion that the cysteine availability is a major determinant for partitioning of cysteine sulfur to either GSH, taurine or inorganic sulfates in liver (Stipanuk et al., 1992). Low cysteine availability would favor its utilization for the synthesis of GSH; high cysteine availability enhances its catabolism to inorganic sulfate and taurine. But in this study hepatic taurine was rather decreased significantly whereas taurine in plasma was increased. It appears that the enhancement of hepatic efflux of taurine is greater than the increase in catabolism of cysteine to taurine in acute liver injury, resulting in reduction of hepatic levels of this $\beta$-amino acid. 
The increase in plasma levels of GSH and taurine is of special interest. Plasma GSH reached a level greater than ninefold of control in $24 \mathrm{~h}$ after $\mathrm{CCl}_{4}$ treatment. Liver has a central role in the maintenance of interorgan GSH homeostasis with sinusoidal GSH efflux as the major determinant of $\mathrm{GSH}$, cystine, cysteine, and thiol-disulfide status of plasma (Ookhtens and Kaplowitz, 1998). The molecular mechanisms of GSH efflux have been suggested to involve several transporters including oatpl (the sinusoidal organic anion transporter polypeptide), gsht (a putative GSH transporter on the sinusoidal side), and multidrug resistance proteins (MRPs). The enhancement of GSH efflux after $\mathrm{CCl}_{4}$ treatment has been demonstrated by several authors (Irita et al., 1994; Kadiiska et al., 2000). Interestingly, the total antioxidant capacity of serum was also increased, but the elevation of GSH could account for only a small portion of the enhanced total antioxidant capacity in serum (Kadiiska et al., 2000). In this study the elevation of plasma GSH does not appear to be associated with inhibition of its catabolism. The metabolism of GSH is mediated by $\gamma$-glutamyltransferase (GGT) that allows component amino acids to be available for intracellular GSH resynthesis and a continuous 'GSH cycling' (Whitfield, 2001). The GGT activity in kidney was not altered by $\mathrm{CCl}_{4}$ treatment, indicating that the accumulation of plasma GSH might not be attributed to the destruction of this enzyme system. The dose of $\mathrm{CCl}_{4}$ used was also nephrotoxic as shown by the elevation of BUN and $\mathrm{sCr}$. The increase in $\mathrm{BUN}$ and $\mathrm{sCr}$ indicated that glomerular filtration rate was decreased in rats treated with $\mathrm{CCl}_{4}$. However, the changes in the plasma concentration of other sulfur-containing metabolites were much smaller, suggesting that the reduction in glomerular filtration rate might not account for the marked elevation of plasma GSH. Further studies need to be conducted to identify the mechanism of GSH elevation in plasma in association with induction of acute liver injury by $\mathrm{CCl}_{4}$.

In summary, the present results indicate that induction of acute liver injury by a toxic dose of $\mathrm{CCl}_{4}$ is accompanied with extensive alterations in the metabolomics of sulfur-containing amino acids and related substances. The increase in hepatic MAT without a change in SAM levels suggests that the utilization of SAM is increased in a reaction other than the transsulfuration reactions. The cellular availability of cysteine and activities of both $\mathrm{CDO}$ and $\mathrm{GCL}$ are elevated, resulting in an increase in GSH and hypotaurine syntheses in liver. The major metabolites and products of the transsulfuration pathway, including methionine, cysteine, hypotaurine or taurine, and GSH, are all increased in liver and plasma after the treatment. The physiological significance of the change in the metabolomics of sulfur-containing substances and its role in the induction of liver injury warrant further studies.

\section{ACKNOWLEDGEMENTS}

This work was supported in part by a Korea Science and Engineering Foundation (KOSEF) grant (R01-2008000-10622-0) funded by the Ministry of Education, Science and Technology, Korea.

\section{REFERENCES}

Adinolfi, L.E., Ingrosso, D., Cesaro, G., Cimmino, A., D'Anto, M., Capasso, R., Zappia, V. and Ruggiero, G. (2005). Hyperhomocysteinemia and the MTHFR C677T polymorphism promote steatosis and fibrosis in chronic hepatitis C patients. Hepatology, 41, 995-1003.

Bagley, P.J., Hirschberger, L.L. and Stipanuk, M.H. (1995). Evaluation and modification of an assay procedure for cysteine dioxygenase activity: High-performance liquid chromatography method for measurement of cysteinesulfinate and demonstration of physiological relevance of cysteine dioxygenase activity in cysteine catabolism. Anal. Biochem., 227, 40-48.

Corrales, F., Giménez, A., Alvarez, L., Caballería, J., Pajares, M.A., Andreu, H., Parés, A., Mato, J.M. and Rodés, J. (1992). S-adenosylmethionine treatment prevents carbon tetrachloride-induced S-adenosylmethionine synthetase inactivation and attenuates liver injury. Hepatology, 16, 10221027.

Corrales, F.J., Ruiz, F. and Mato, J.M. (1999). In vivo regulation by glutathione of methionine adenosyltransferase $S$ nitrosylation in rat liver. J. Hepatol., 31, 887-894.

Gaitonde, M.K. (1967). A spectrophotometric method for the direct determination of cysteine in the presence of other naturally occurring amino acids. Biochem. J., 104, 627-633.

Griffith, O.W. (1980). Determination of glutathione and glutathione disulfide using glutathione reductase and 2vinylpyridine. Anal. Biochem., 106, 207-212.

Hinchman, C.A. and Ballatori, N. (1999). Glutathione-degrading capacities of liver and kidney in different species. Biochem. Pharmacol., 40, 1131-1135.

Horowitz, J.H., Rypins, E.B., Henderson, J.M., Heymsfield, S.B., Moffitt, S.D., Bain, R.P., Chawla, R.K., Bleier, J.C. and Rudman, D. (1981). Evidence for impairment of transsulfuration pathway in cirrhosis. Gastroenterology, 81, 668675.

Ide, T. (1997). Simple high-performance liquid chromatographic method for assaying cysteinesulfinic acid decarboxylase activity in rat tissue. J. Chromatogr. B Biomed. Sci. Appl., 694, 325-332.

Irita, K., Okabe, H., Koga, A., Kurosawa, K., Tagawa, K., Yamakawa, M., Yoshitake, J. and Takahashi, S. (1994). Carbon tetrachloride increases sinusoidal efflux of reduced and oxidized glutathione in rats. Biochem. Pharmacol., 47, 
447-452.

Ji, C., Deng, Q. and Kaplowitz, N. (2004). Role of TNF-alpha in ethanol-induced hyperhomocysteinemia and murine alcoholic liver injury. Hepatology, 40, 442-451.

Kadiiska, M.B., Gladen, B.C., Baird, D.D., Dikalova, A.E., Sohal, R.S., Hatch, G.E., Jones, D.P., Mason, R.P. and Barrett, J.C. (2000). Biomarkers of oxidative stress study: are plasma antioxidants markers of $\mathrm{CCl}_{4}$ poisoning? Free Radic. Biol. Med., 28, 838-845.

Kashiwamata, S. and Greenberg, D.M. (1970). Studies on cystathionine synthase of rat liver. Properties of the highly purified enzyme. Biochim. Biophys. Acta, 212, 488-500.

Kim, S.J., Jung, Y.S., Kwon, D.Y. and Kim, Y.C. (2008). Alleviation of acute ethanol-induced liver injury and impaired metabolomics of S-containing substances by betaine supplementation. Biochem. Biophys. Res. Commun., 368, 893898.

Kim, S.K. and Kim, Y.C. (2002). Attenuation of bacterial lipopolysaccharide-induced hepatotoxicity by betaine or taurine in rats. Food Chem. Toxicol., 40, 545-549.

Kim, S.K. and Kim, Y.C. (2005). Effects of betaine supplementation on hepatic metabolism of sulfur-containing amino acids in mice. J. Hepatol., 42, 907-913.

Kim, Y.C., Jung, Y.S. and Kim, S.K. (2005). Effect of betaine supplementation on changes in hepatic metabolism of sulfur-containing amino acids and experimental cholestasis induced by alpha-naphthylisothiocyanate. Food Chem. Toxicol., 43, 663-670.

Kinsell, L.W., Harper, H.A., Barton, H.C., Michaels, G.D. and Weiss, H.A. (1947). Rate of disappearance from plasma of intravenously administered methionine in patients with liver damage. Science, 106, 589-594.

Kwon, D.Y., Jung, Y.S., Kim, S.J., Park, H.K., Park, J.H. and Kim, Y.C. (2008). Impaired sulfur-amino acid metabolism and oxidative stress in non-alcoholic fatty liver are alleviated by betaine supplementation in rats. J. Nutr. (in press).

Lee, S.Y. and Kim, Y.C. (2007). Effect of beta-alanine administration on carbon tetrachloride-induced acute hepatotoxicity. Amino Acids, 33, 543-546.

Lu, S.C. (1998). Regulation of hepatic glutathione synthesis. Semin. Liver Dis., 18, 331-343.

Mato, J.M. and Lu, S.C. (2007). Role of S-adenosyl-L-methionine in liver health and injury. Hepatology, 45, 1306-1312.

Matsuo, Y. and Greenberg, D.M. (1957). A crystalline enzyme that cleaves homoserine and cystathionine. I. isolation procedure and some physicochemical properties. J. Biol. Chem., 230, 545-560.

Miyazaki, T., Karube, M., Matsuzaki, Y., Ikegami, T., Doy, M., Tanaka, N. and Bouscarel, B. (2005). Taurine inhibits oxidative damage and prevents fibrosis in carbon tetrachloride-induced hepatic fibrosis. J. Hepatol., 43, 117-125.

Mudd, S.H. and Poole, J.R. (1975). Labile methyl balances for normal humans on various dietary regimens. Metabolism, 24, 721-735.

Nieto, N. and Cederbaum, A.I. (2005). S-adenosylmethionine blocks collagen I production by preventing transforming growth factor-beta induction of the COL1A2 promoter. $\mathrm{J}$. Biol. Chem., 280, 30963-30974.
Ookhtens, M. and Kaplowitz, N. (1998). Role of the liver in interorgan homeostasis of glutathione and cyst(e)ine. Semin. Liver Dis., 18, 313-329.

Orlowski, M. and Meister, A. (1963). $\gamma$-Glutamyl-p-nitroanilide: a new convenient substrate for determination and study of $\mathrm{L}$ - and D-glutamyltranspeptidase activities. Biochim. Biophys. Acta, 73, 676-679.

Pajares, M.A., Durán, C., Corrales, F., Pliego, M.M. and Mato, J.M. (1992). Modulation of rat liver S-adenosylmethionine synthetase activity by glutathione. J. Biol. Chem., 267, 17598-17605.

Rajendra, W. (1987). High performance liquid chromatographic determination of amino acids in biological samples by precolumn derivatization with O-phthaldehyde. J. Liq. Chromatogr. Relat. Technol., 10, 941-955.

Recknagel, R.O., Glende, Jr., E.A., Dolak, J.A. and Waller, R.L. (1989). Mechanisms of carbon tetrachloride toxicity. Pharmacol. Ther., 43, 139-154.

Reitman, S. and Frankel, S.A. (1957). Colorimetric method for determination of serum glutamic oxaloacetic and glutamic pyruvic transaminases. Am. J. Clin. Pathol., 28, 56-63.

Sánchez-Góngora, E., Ruiz, F., Mingorance, J., An, W., Corrales, F.J. and Mato, J.M. (1997). Interaction of liver methionine adenosyltransferase with hydroxyl radical. FASEB J., 11, 1013-1019.

Sekura, R. and Meister, A. (1977). Gamma-Glutamylcysteine synthetase. Further purification, "half of the sites" reactivity, subunits, and specificity. J. Biol. Chem., 252, 25992605.

She, Q.B., Nagao, I., Hayakawa, T. and Tsuge, H. (1994). A simple HPLC method for the determination of S-adenosylmethionine and S-adenosylhomocysteine in rat tissues: the effect of vitamin B6 deficiency on these concentrations in rat liver. Biochem. Biophys. Res. Commun., 205, 17481754.

Simile, M.M., Banni, S., Angioni, E., Carta, G., De Miglio, M.R., Muroni, M.R., Calvisi, D.F., Carru, A., Pascale, R.M. and Feo, F. (2001). 5'-Methylthioadenosine administration prevents lipid peroxidation and fibrogenesis induced in rat liver by carbon-tetrachloride intoxication. J. Hepatol., 34, 386-394.

Stipanuk, M.H., Coloso, R.M., Garcia, R.A. and Banks, M.F. (1992). Cysteine concentration regulates cysteine metabolism to glutathione, sulfate and taurine in rat hepatocytes. J. Nutr., 122, 420-427.

Volpi, N. and Tarugi, P. (1998). Improvement in the high-performance liquid chromatography malondialdehyde level determination in normal human plasma. J. Chromatogr. $B$ Biomed. Sci. Appl., 713, 433-437.

Whitfield, J.B. (2001). Gamma glutamyl transferase. Crit. Rev. Clin. Lab. Sci., 38, 263-355.

Yan, C.C. and Huxtable, R.J. (1995). Fluorimetric determination of monobromobimane and O-phthalaldehyde adducts of gamma-glutamylcysteine and glutathione: application to assay of gamma-glutamylcysteinyl synthetase activity and glutathione concentration in liver. J. Chromatogr. B Biomed. Appl., 672, 217-224. 